\title{
Experimental Results on Hadronic b Decays
}

\section{Y. Kubota}

University of Minnesota, Minneapolis, Minnesota, 55455 USA

E-mail: 'yk@mnhep.hep.umn.edü

Abstract: The current experimental status of hadronic decays of $b$ hadrons is reviewed. The topics discussed are: tests of the factorization hypothesis, measurements of $B$ meson decays to one of the charmonia states and a "K" meson, measurements of the number of $D$ mesons arising from $W$ vertices and the observation of a new charm meson $D_{1}^{0}\left(j=\frac{1}{2}\right)$ in exclusive decays of the $B$.

\section{Tests of Factorization Hypothesis and Final-State Interactions}

It is common to assume factorization when the rate for a hadronic decay of a $B$ meson is calculated. However, it is desirable to test it experimentally. This can be done by comparing the helicity amplitudes of proper hadronic and semileptonic decays.

Even if factorization is valid, if there are significant contributions from final-state interactions (FSI) in $B$ decays, they may manifest as nontrivial relative phases among different helicity amplitudes.

Recently, the CLEO experiment measured the helicity amplitudes of the decay $B \rightarrow D^{*} \rho^{-}$ where both the $B$ and $D^{*}$ can be either charged or neutral. They tested the factorization hypothesis and looked for effects of FSI using the method described above.

If factorization is valid, the amplitudes of $B \rightarrow D^{*} \rho^{-}$should be proportional to the amplitudes for the semileptonic decay $B \rightarrow D^{*} \ell \nu$ at the momentum transfer value, $q^{2}=m_{\rho}^{2}$. However, since no experiments have measured individual helicity amplitudes for the semileptonic decay at this particular $q^{2}$ value, they made a comparison of the longitudinal polarization, $\Gamma_{L} / \Gamma=$ $\left|H_{0}\right|^{2} / \sum\left|H_{i}\right|^{2}$, of the $D^{*}$ for the two decays [i]

Figure 1'ishows the mass distributions of CLEO $B \rightarrow D^{*} \rho^{-}$candidates in the two charge modes. Clear peaks for the signal are seen over a small background. Since the decays are fully recon- structed, they can measure all three decay angles, namely the $D^{*}$ and $\rho^{-}$decay angles measured in their rest frames relative to the direction of the $B$ as well as the angle between the two decay planes, as illustrated in figure
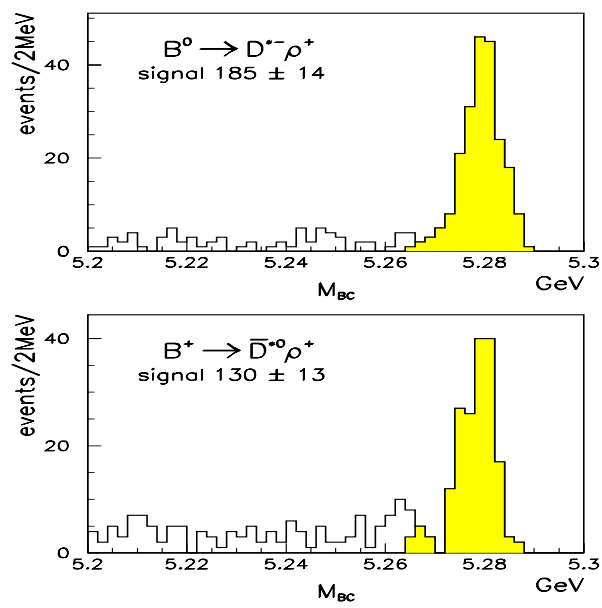

Figure 1: Distributions of $B$ candidate masses for $B^{0}$ and $B^{+}$.

They analysed the three dimensional distribution of these angles using a maximum likelihood method and obtained the helicity amplitudes. Figure $\overline{\beta_{1}}$, shows the projections of the fit results on the distributions of the three angles of $\bar{B}^{0} \rightarrow D^{*+} \rho^{-}$candidates. The resulting helicity amplitudes for each of the two charge modes are listed in table $\overline{1}$.'.

From these results, the longitudinal polarization was calculated to be $\Gamma_{L} / \Gamma=0.878 \pm$ $0.034 \pm 0.040$. This agrees well with the po- 


\begin{tabular}{|l|cc|}
\hline $\bar{B}^{0} \rightarrow D^{*+} \rho^{-}$ & Magnitude & Phase (rad) \\
\hline$H_{0}$ & 0.936 & 0 by definition \\
\hline$H_{-}$ & $0.317 \pm 0.052 \pm 0.013$ & $0.19 \pm 0.23 \pm 0.14$ \\
\hline$H_{+}$ & $0.152 \pm 0.058 \pm 0.037$ & $1.47 \pm 0.37 \pm 0.32$ \\
\hline \hline$B^{-} \rightarrow D^{* 0} \rho^{+}$ & Magnitude & Phase (rad) \\
\hline$H_{0}$ & 0.932 & 0 by definition \\
\hline$H_{-}$ & $0.283 \pm 0.068 \pm 0.039$ & $1.13 \pm 0.27 \pm 0.17$ \\
\hline$H_{+}$ & $0.228 \pm 0.069 \pm 0.036$ & $0.95 \pm 0.31 \pm 0.19$ \\
\hline
\end{tabular}

Table 1: Helicity amplitudes measured for $\bar{B}^{0} \rightarrow D^{*+} \rho^{-}$and $B^{-} \rightarrow D^{* 0} \rho^{-}$

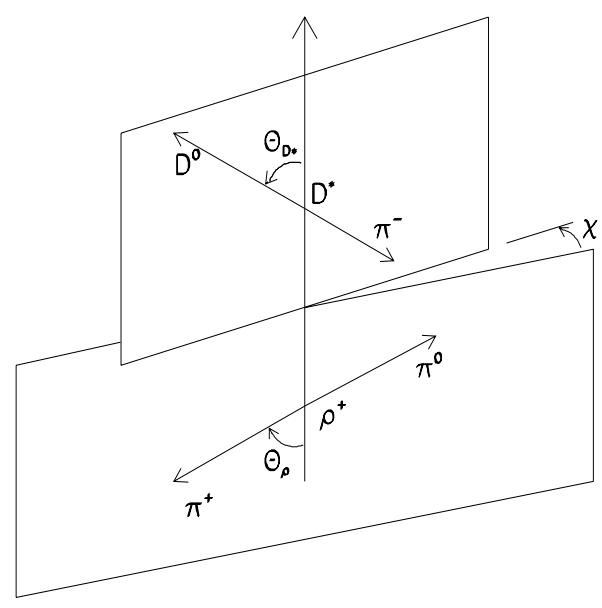

Figure 2: Definition of the decay angles of $B \rightarrow$ $D^{*} \rho^{-}$decay.
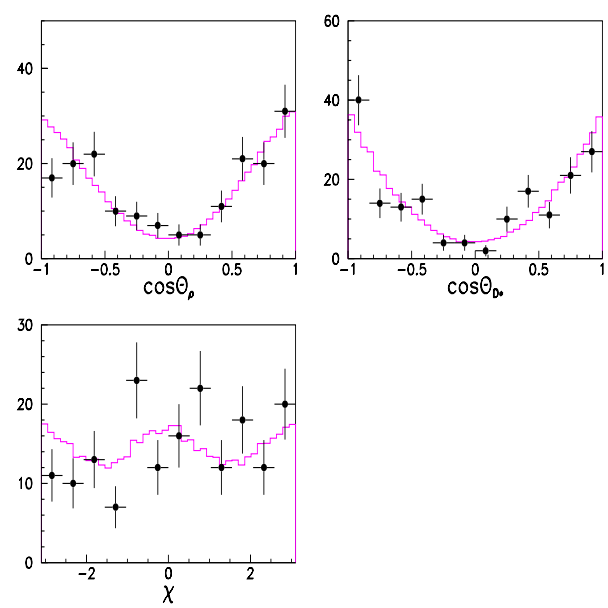

Figure 3: Decay angle distributions of $\bar{B}^{0} \rightarrow$ $D^{*+} \rho^{-}$decay.

larization measured for the semileptonic decay, $0.914 \pm 0.152 \pm 0.089[\overrightarrow{2}]$, and also the theoretical expectation, $0.85-0.88$ [3i] cates that factorization is a reasonable assump-

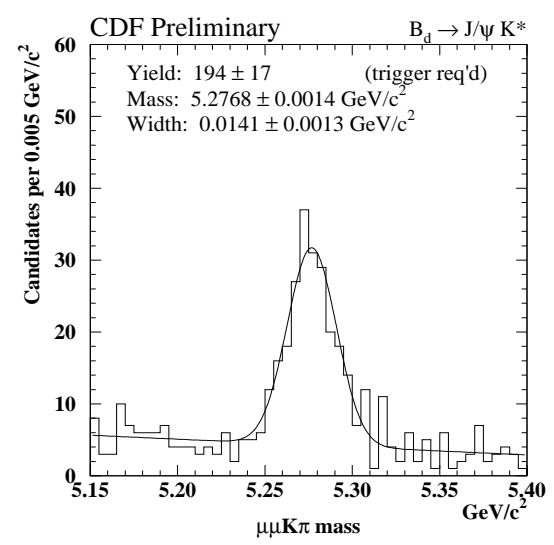

Figure 4: Mass distribution of $B \rightarrow(J / \psi) K^{*}$ candidates.

tion in $B$ hadronic decays.

The relative phases of the helicity amplitudes, on the other hand, are somewhat different from zero. This implies that FSI may play a role even in $B$ decays against prejudice. However, we should wait before drawing more definitive conclusions from these results until the full data set is analysed, in particular with regard to the effects of the background.

A similar analysis was carried out by the CDF collaboration for the decays $B \rightarrow(J / \psi) K^{*}$ and $\left.B_{S} \rightarrow(J / \psi) \phi[]_{1}^{4}\right]$. The distributions of the masses of the candidates in figures $\overline{4}_{1}^{\prime}$ and $\overline{1}$, clear signals.

The results are expressed in terms of transversity amplitudes, instead of the helicity amplitudes, and are listed in table $\overline{2}_{r}^{\prime}$ It is done this way because two of the amplitudes represent states of one parity (and CP parity) and the third represents the opposite parity state. These need to 


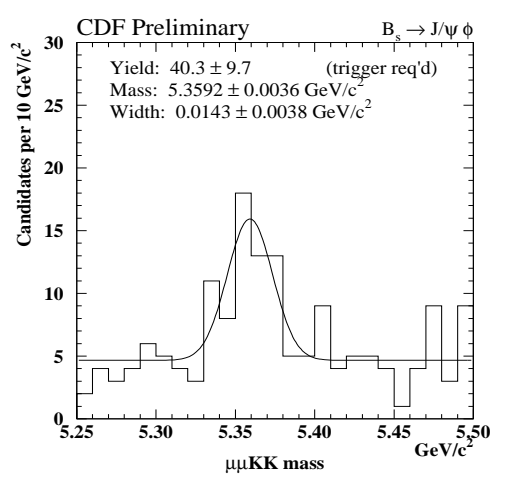

Figure 5: Mass distribution of $B \rightarrow(J / \psi) \phi$ candidates.

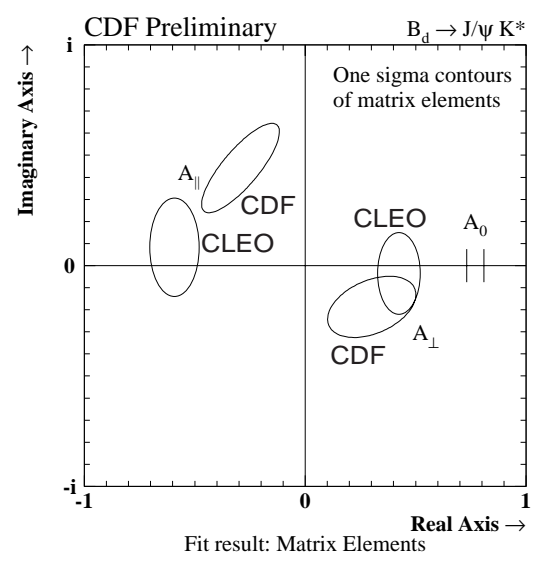

Figure 6: Transversity amplitudes of the $B \rightarrow$ $(J / \psi) K^{*}$ decay.

be distinguished if these decays are to be used to study $\mathrm{CP}$ violation phenomena. These results

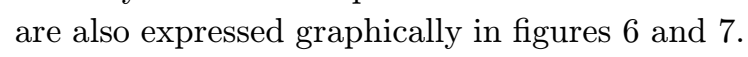

Though there are no proper semileptonic decays to test factorization for these decays, the relative phase measurements are still useful to explore effects of FSI. The non-zero phases of these measurements for $B \rightarrow(J / \psi) K^{*}$ imply that FSI plays a role in this decay mode. Previous results from CLEO for the $B$ decay $[\overline{5}$, however, are consistent with no phase differences among the three amplitudes. The two sets of results are in fact statistically consistent though they give a qualitatively different impression. We therefore need to wait for more precise results from

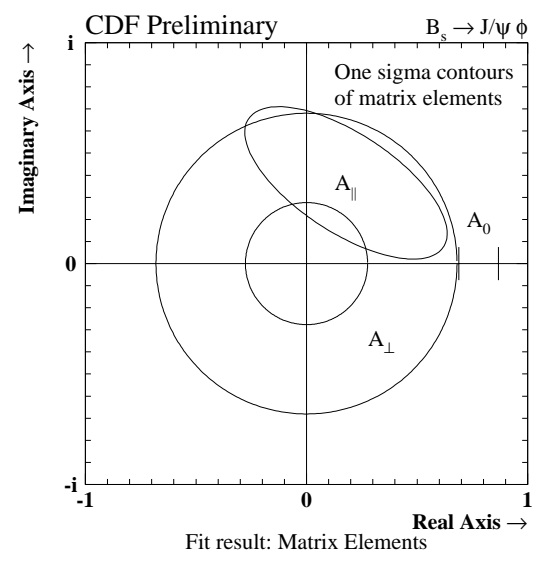

Figure 7: Transversity amplitudes of the $B \rightarrow$ $(J / \psi) \phi$ decay.

future experiments such as CLEO (which have tripled their data since their previous measurements were made) and other B-factory experiments. A measure of the CP-odd component, $\left|A_{\perp}\right|^{2}=\frac{\Gamma_{\perp}}{\Gamma}$, can be calculated from these results and is $0.126_{-0.093}^{+0.121} \pm 0.028$, which is consistent with CLEO. The longitudinal polarization, $\left|A_{\|}\right|^{2}=\frac{\Gamma_{L}}{\Gamma}$, can also be obtained and is consistent with the previous results from CLEO and CDF's RUN IA data.

The first result on $\left|A_{\perp}\right|^{2}=\frac{\Gamma_{\perp}}{\Gamma}$ for $B_{S} \rightarrow$ $(\mathrm{J} / \psi) \phi$ is $0.229 \pm 0.188 \pm 0.038$ and is consistent with zero. Their new value for $\frac{\Gamma_{L}}{\Gamma}$ of $0.606 \pm$ $0.139 \pm 0.018$ is consistent with their previous result.

\section{2. $B$ to Charmonium Exclusive De- cays}

It is very useful to explore pure CP decays of $B$ mesons involving charmonia beside the famous $B \rightarrow(J / \psi) K_{S}^{0}$ decay. The decays CDF observed and described above are examples (when $K^{* 0} \rightarrow$ $\left.K_{S}^{0} \pi^{0}\right)$. In addition, the CLEO group has observed $B \rightarrow \psi(2 S) K^{*}, B \rightarrow(J / \psi) K_{L}^{0}$ using its CsI calorimeter, $B \rightarrow(J / \psi) \pi^{0}$ and $B \rightarrow \chi_{c 1} K_{S}^{0}$ decays. Figures $\bar{l}_{-1}$ and ${ }_{9}^{\overline{9}}$ illustrate how well these decays are reconstructed.

Meanwhile, they have improved the efficiencies for detecting $J / \psi \rightarrow \mu \mu$ and ee decays by about $20 \%$. They did so by (1) identifying some of the muons in the calorimeter, and (2) looking 


\begin{tabular}{|l|cc|}
\hline$B \rightarrow(J / \psi) K^{*}$ & Magnitude & Phase (rad) \\
\hline$A_{0}$ & $0.770 \pm 0.039 \pm 0.012$ & 0 by definition \\
\hline$A_{\|}$ & $0.530 \pm 0.106 \pm 0.034$ & $2.16 \pm 0.46 \pm 0.10$ \\
& & $3.00 \pm 0.37 \pm 0.04$ (CLEO) \\
\hline$A_{\perp}$ & $0.355 \pm 0.156 \pm 0.039$ & $-0.56 \pm 0.53 \pm 0.12$ \\
& & $-0.11 \pm 0.46 \pm 0.03$ (CLEO) \\
\hline$\Gamma_{\perp} / \Gamma=\left|A_{\perp}\right|^{2}$ & $0.126_{-0.093}^{+0.121} \pm 0.028$ & \\
& $0.16 \pm 0.08 \pm 0.04(\mathrm{CLEO})$ & \\
\hline$\Gamma_{L} / \Gamma=\left|A_{\|}\right|^{2}$ & $0.593_{-0.061}^{+0.059} \pm 0.018$ & \\
& $0.52 \pm 0.07 \pm 0.04(\mathrm{CLEO})$ & \\
& $0.65 \pm 0.10 \pm 0.04(\mathrm{RUN} \mathrm{IA})$ & \\
\hline \hline$B_{S} \rightarrow(J / \psi) \phi$ & Magnitude & Phase (rad) \\
\hline$A_{0}$ & $0.778 \pm 0.090 \pm 0.012$ & 0 by definition \\
\hline$A_{\|}$ & $0.407 \pm 0.232 \pm 0.034$ & $1.12 \pm 1.29 \pm 0.11$ \\
\hline$A_{\perp}$ & $0.478 \pm 0.202 \pm 0.040$ & no sensitivity \\
\hline$\Gamma_{\perp} / \Gamma=\left|A_{\perp}\right|^{2}$ & $0.229 \pm 0.188 \pm 0.038$ & \\
\hline$\Gamma_{L} / \Gamma=\left|A_{\|}\right|^{2}$ & $0.606 \pm 0.139 \pm 0.018$ & \\
& $0.56 \pm 0.21_{-0.04}^{+0.02}(\mathrm{RUN}$ IA) & \\
\hline
\end{tabular}

Table 2: Transversity amplitudes measured for $B \rightarrow(J / \psi) K^{*}$ and $B_{S} \rightarrow(J / \psi) \phi$. There is no sensitivity to the phase of $A_{\perp}$ for $B_{S} \rightarrow(J / \psi) \phi$ due to cancellation of its contribution in $B_{S}$ and $\bar{B}_{S}$, which cannot be distinguished in this decay mode.

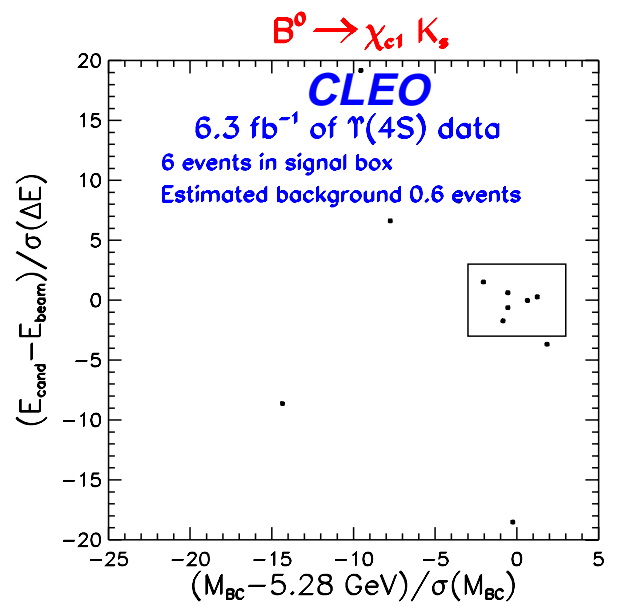

Figure 8: Mass distribution of candidate $B \rightarrow$ $\chi_{c 1} K_{S}^{0}$ decays.

for $J / \psi \rightarrow e^{+} e^{-} \gamma$, where the $\gamma$ arises from either QED radiation process or when one of the daughter electrons radiates while passing through the detector material.

The distributions of the $\psi \rightarrow \mu \mu$ and $e e$ candidates in figures $\overline{1} \overline{0}_{1}^{\prime}$ and $\overline{1}^{1} \overline{1}_{1}^{1}$ show the improvements in their detection. Table summarizes the increase in the number of decays one can ex-

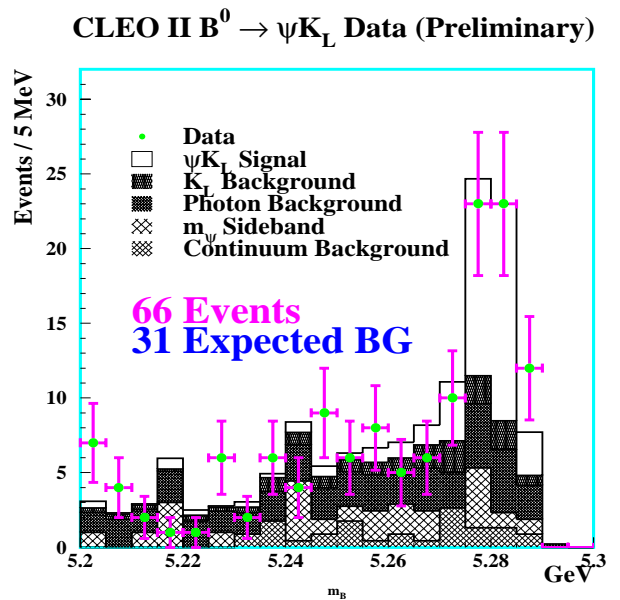

Figure 9: Mass distribution of candidate $B \rightarrow$ $(J / \psi) K_{L}^{0}$ decays.

pect from these decays compared to the golden decay, $B \rightarrow(J / \psi) K_{S}^{0}$.

\section{Wrong-Flavour $D$ from $B$}

It is important to measure the average number of charm particles in $B$ decays for two reasons. We want to know if $b \rightarrow c$ is really $100 \%$ (except small $b \rightarrow u$ contributions). If not, it suggests 


\begin{tabular}{|l|c|c|c|}
\hline Mode & $\begin{array}{c}\text { Events } \\
\text { observed }\end{array}$ & $C P$ & Branching Ratio \\
\hline \hline$B^{0} \rightarrow J / \psi K^{0}$ & & & \\
..$K_{S} \rightarrow \pi^{+} \pi^{-}$ & 75 & -1 & $(0.92 \pm 0.11 \pm 0.11) \cdot 10^{-3}$ \\
..$K_{S} \rightarrow \pi^{0} \pi^{0}$ & 15 & -1 & $(1.21 \pm 0.31 \pm 0.25) \cdot 10^{-3}$ \\
..$K_{L}$ in calorimeter & 35 & +1 & \\
\hline$B^{0} \rightarrow J / \psi \pi^{0}$ & 7 & +1 & $\left(3.4_{-1.5}^{+1.7} \pm 0.4\right) \cdot 10^{-5}$ \\
\hline$B^{0} \rightarrow J / \psi \eta$ & 0 & +1 & $<5.8 \cdot 10^{-5} 90 \%$ C.L. \\
\hline$B^{0} \rightarrow \chi_{c 1} K_{S}^{0}$ & 6 & -1 & $\left(4.5_{-1.8}^{+2.8} \pm 0.9\right) \cdot 10^{-4}$ \\
\hline$B^{0} \rightarrow \psi(2 S) K_{S}^{0}$ & 15 & -1 & $(5.2 \pm 1.4 \pm 0.5) \cdot 10^{-4}$ \\
\hline
\end{tabular}

Table 3: Number of events reconstructed in various $B$ decay modes involving charmonia.

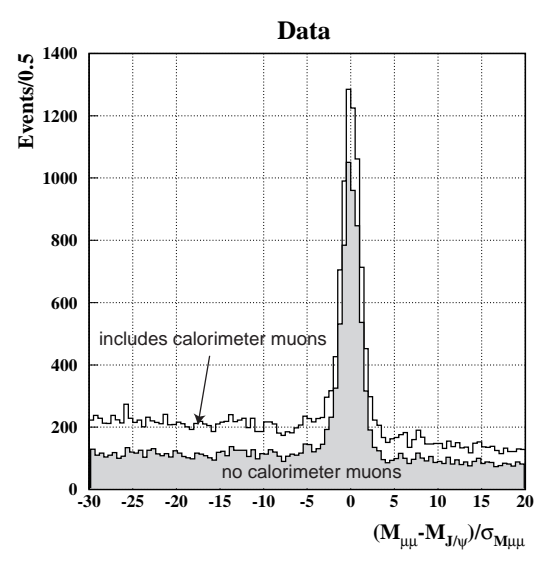

Figure 10: Mass distribution of $\psi \rightarrow \mu \mu$ decay candidates using muons identified with the calorimeter.

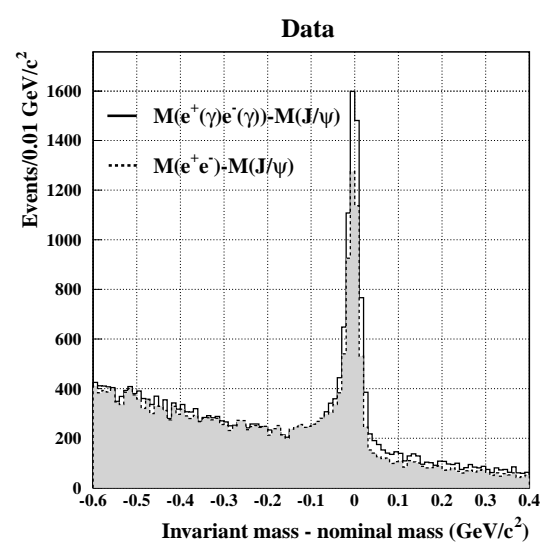

Figure 11: Mass distribution of $\psi \rightarrow e e$ decay candidates, including those with additional photons.

that there are, for example, significant hadronic penguin contributions in hadronic $B$ decays. Sec- ond, we would like to know how often charm particles arise from $W$ decays $W \rightarrow \bar{c} s$. Theories predict that the rate is about 15 to $30 \%$, but the calculation is not as reliable as for $W \rightarrow \bar{u} d$. The uncertainty in this rate dominates the uncertainty in the rate of $W \rightarrow$ hadrons, and therefore critical in the calculation of $\mathcal{B}_{S L}=\Gamma_{S L} /\left(\Gamma_{S L}+\right.$ $\left.\Gamma_{h a d}\right)$. Considering the long standing problem with the lower-than expected semileptonic branching fraction, it is imperative that we measure the rate for $W \rightarrow \bar{c} s$. Experiments have accomplished this by either (1) making flavour specific measurements of charm production rates $(B \rightarrow$ $D$ or $\bar{B} \rightarrow \bar{D}$ arising from $W \rightarrow \bar{c} s$ as opposed to $B \rightarrow \bar{D}$ or $\bar{B} \rightarrow D$ arising from $b \rightarrow c W$ ) the double-charm decays of the $B$; or (2) measuring the rate for double-charm production $B \rightarrow$ $\bar{D} D X$.

The inclusive charm production rate in $B$ decays (sum of charm and anti-charm) has been measured to be somewhat lower than what we expected. More recent measurements of charm multiplicities are higher, and the ALEPH experiment, for example, measures it to be $1.23 \pm 0.08$.

The CLEO experiment measured flavour specific charm multiplicities charm particles arising from the virtual $W(W \rightarrow$ $\bar{c} s$ ) and those which come from $b \rightarrow c W$ separately. The upper limits for non-charm productions in hadronic $B$ decays (such as $B \rightarrow$ gluon + strange particle) was determined from the number of charm particles observed in hadronic $B$ decays with the proper flavour for $b \rightarrow c W$. In this measurement, since you are interested in showing how close to $100 \%$ the charm production in $B$ de- 
cays is, it was important to know the detection efficiencies and the decay branching fractions of the charm particles very well. This was accomplished using the number of charm particles in semileptonic $B$ decays, where we know that the charm particles production is close to $100 \%$ to a high accuracy. This is because semileptonic $B$ decays are either $b \rightarrow c \ell \nu$ producing one charm particle or $b \rightarrow u \ell \nu$ producing no charm particle, and we know the ratio between their rates. CLEO observed a little fewer charm particles than expected for semileptonic $B$ decays, but it was still consistent within the error. This confirmed that their estimates of charm detection efficiencies and current measured values of the charm decay branching fractions were reasonable within their quoted errors.

They found that the $D$ meson are produced from the $W 7.9 \pm 2.2 \%$ of the time, more copiously than naively thought previously. In addition, they found $B \rightarrow D_{S} 10.0 \pm 2.7 \%$ of the time, $B \rightarrow$ charmonia $3.0 \pm 0.5 \%$ of the time and charm baryons $1.0 \pm 0.6 \%$ of the time. They amount to a total of $22 \pm 4 \%$ of charm particles from the $W$. This is consistent with our expectation. The possible charm deficit observed in the inclusive charm production rate was then attributed to the systematic uncertainties in counting charm particles from $b \rightarrow c W$. Currently, it is consistent with unity within the systematic error as described above.

Recently, the ALEPH, DELPHI and SLD experiments measured the fractions of doublecharm $B$ decays to confirm the CLEO observation on $W \rightarrow \bar{c} s$. ALEPH [isi] took advantage of the fact that double-charm $B$ decays tend to contain more events with two identified vertices away from the primary interaction point. Figure ${ }_{1}^{1} \overline{2}_{1}^{1}$ shows an example of how multiple vertices are found. Figure 13 shows the distance between the two vertices $\left(\bar{d}_{B D}\right)$ normalized to the resolution. It demonstrates that doubly-charmed events have a significant separation between the two vertices. By analysing these distributions, they found that

$$
\begin{aligned}
& \mathcal{B}\left(b \rightarrow D_{s} D X\right)= \\
& 13.1_{-2.2}^{+2.6}(\text { stat })_{-1.6}^{+1.8}(\text { sys })_{-2.7}^{+4.4}\left(\mathcal{B}_{D}\right) \%,
\end{aligned}
$$

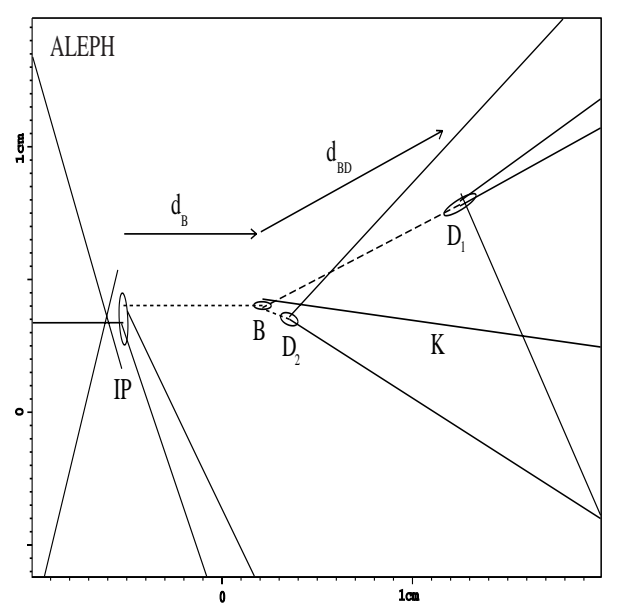

Figure 12: Reconstructed vertices of a $B$ decay candidate from ALEPH.
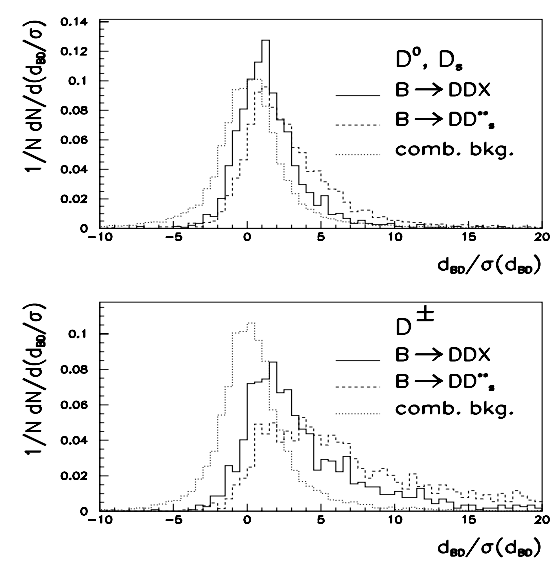

Figure 13: Vertex separation distribution of $B$ decay candidates from ALEPH.

and

$$
\begin{aligned}
& \mathcal{B}(b \rightarrow \bar{D} D X)= \\
& \quad 7.8_{-1.8}^{+2.0}(\text { stat })_{-1.5}^{+1.7}(\text { sys })_{-0.4}^{+0.5}\left(\mathcal{B}_{D}\right) \% .
\end{aligned}
$$

Since their results are for a mixture of $b$ hadrons, these results cannot be directly compared with the CLEO results. If one assumes, however, that there are no $\bar{D} D X$ decays other than $B_{d}$ and $B_{u}$, the latter number will be translated into

$$
\begin{aligned}
& \mathcal{B}(B \rightarrow \bar{D} D X)= \\
& \quad 10.3_{-2.4}^{+2.6}(\text { stat })_{-2.0}^{+2.2}(\text { sys })_{-0.5}^{+0.7}\left(\mathcal{B}_{D}\right) \%
\end{aligned}
$$

which is consistent with the CLEO results of $(7.9 \pm 2.2) \%$. 


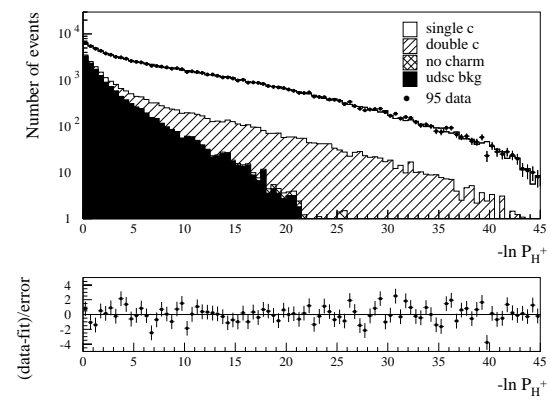

Figure 14: negative log likelihood, $-\ln P$, for a $b$ jet tracks to have come from a single vertex (DELPHI). They look for events with small likelihood (large $-\ln P$ ), which presumably come from singleor double-charm $b$-hadron decays producing multiple vertices.

The DELPHI experiment also looked for doublecharm decays of $b$ hadrons by how well $b$ events are consistent with having only one decay ver-

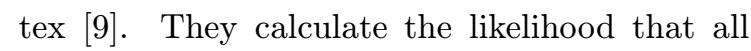
tracks in a $b$ jet come from the a single vertex. Events with one or two charm particles tend to have smaller likelihood. They show a distribution of negative-log likelihood, $-\ln P$, which I reproduce in figure 14. The long tail seen in the graph is due to single- and double-charm events. From this analysis, they obtain

$$
\mathcal{B}_{0 C}=(3.3 \pm 2.1) \%,
$$

and

$$
\mathcal{B}_{2 C}=(13.6 \pm 4.2) \% .
$$

The latter result include all charm particle (unlike the ALEPH results described above) and is consistent with the value obtained by CLEO (19\%土 but somewhat lower. By subtracting the charmonia contributions from the "no-charm" yield, they set an upper limit on the charmless decays of $3.7 \%$.

The analysis of the SLD experiment has a little twist to that of DELPHI. They select those events with two identified decay vertices. This sample is mainly a mixture of single- and doublecharm $b$-hadron decays. Then they look for the numbers of kaons from the $b$ - and $c$-decay vertices. In single-charm decays, what they identify as the $b$-decay vertex should not contain many kaons and $c$ vertex must contain typically one kaon. In double-charm decays, what they identify as the $b$-decay vertex may contain kaons from one of the two charm decays. They expect that the ratio of the numbers of kaons from doublecharm events is $1.270 \pm 0.026$ and that for other events is $0.517 \pm 0.007$. In data, they find this ratio to be $0.688 \pm 0.020$. After applying a few corrections, they obtain a preliminary result for the double-charm branching fraction [1] 1001$]$ :

$$
\mathcal{B}_{2 C}=(16.2 \pm 1.9(\text { stat }) \pm 4.2(\text { sys })) \% .
$$

This is consistent with the CLEO result of $19 \%$. The probability that a track is assigned to an incorrect vertex is the most serious source of systematic error and it is calibrated using leptons. They use a flavour-tagged $b$ sample and look for leptons of "right" charge (expected for $b$ decays) "wrong" charge (expected for $c$ decays). They then check how often they are associated with $b$ and $c$ vertices.

Given the rather high rate of $B \rightarrow \bar{D} D$ decays, CLEO looked for and found a double-charm decay, $B \rightarrow D^{*+} D^{*-}\left[i_{1}^{1} \overline{1}_{1}^{1}\right.$. This decay does not account for a large portion of the double-charm decays of $B$ mesons. However, it is significant since it will be useful to study CP symmetry in the $B$ decays in future $B$ factories.

Figure $11 \overline{5}_{-1}^{1}$ shows clear signal of 4 events in the signal region where they expect the decay to populate. They expect 0.31 events from various background processes. The corresponding branching fraction is $\left(6.2_{-2.9}^{+4.0}(\right.$ stat $) \pm 1.0($ sys $\left.)\right) \times$ $10^{-4}$. They expect that this branching fraction should be about $\left(f_{D^{*}} / f_{D_{S}^{*}}\right)^{2} \tan ^{2} \theta_{C} \mathcal{B}\left(B^{0} \rightarrow\right.$ $\left.4 \% D_{S}^{*+} D^{*-}\right) \sim 0.1 \%$, which agrees well with the observation.

\section{4. $B$ decay to a new charm meson $D_{1}$}

Two of the four p-wave charm mesons $D_{1}(2420)$ and $D_{2}(2460)$ with relatively narrow decay widths (about $20 \mathrm{MeV}$ ) have been seen in $B$ decays as well as in other charm productions. However, 


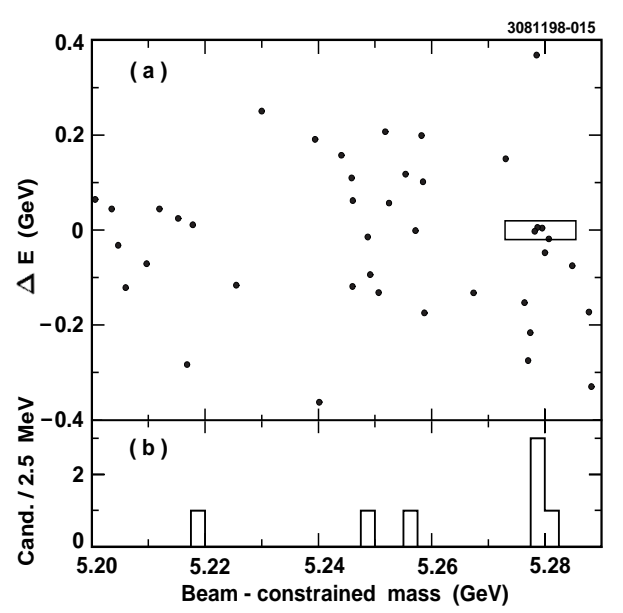

Figure 15: (a) The $\Delta E$ vs the beam-energyconstrained mass distribution for all $B^{0} \rightarrow D^{*+} D^{*-}$ candidates. The signal region is indicated by the box with the solid line. There are four candidates in the signal region and a total of 41 candidates in the entire distribution. (b) The beam-energy-constrained mass distribution for $B^{0} \rightarrow D^{*+} D^{*-}$ candidates satisfying $|\Delta E|<20 \mathrm{MeV}$.

since the other two are expected to have much wider decay widths, it is quite difficult to observe.

CLEO has found one of the two unseen pwave charm meson in their sample of $B^{-} \rightarrow$ $D^{*+} \pi^{-} \pi^{-}$decays [1 ${ }^{\prime}$ ]. The sample was selected using so-called "partial reconstruction method" where the $D^{0}$ decay products were not explic'itly reconstructed in the détector. Since the $\overline{Q-}$ values of the $\bar{D}^{*} \rightarrow \bar{D}^{0} \pi^{+}$and $\bar{\Upsilon}(\bar{S}) \rightarrow \bar{B} \bar{B}$ decays are small, one can infer the momentum of the $D^{0}$ well enough to reconstruct the entire decay chain. They observed about $800 \mathrm{~B}^{-} \rightarrow$ $D^{*+} \pi^{-} \pi^{-}$decays over about 9,000 background events from both continuum $q \bar{q}$ production and other $B$ decays. Partial-wave analysis of about $630 \pm 80 B^{-} \rightarrow D^{*+} \pi^{-} \pi^{-}$decays yielded 344 $B^{-} \rightarrow D_{1}^{0}\left(j=\frac{1}{2}\right) \pi^{-}$decays in addition to 100 $\mathrm{I}^{--} \rightarrow D_{2}^{*}(2460) \bar{\pi}^{-\overline{a n d}^{-}} 223 B^{--} \rightrightarrows D_{1}(2420) \bar{\pi}^{--}$

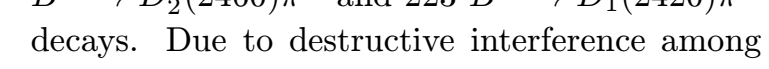
, the three-decay modes, the acturat observed num=

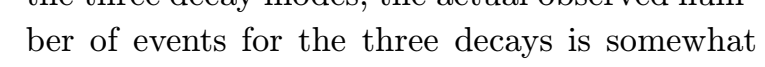
- sraaller-than the sum- of-events-attributed-tø-the

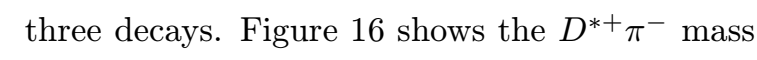
distributions weighing each event by weight based on the decay angles to enhance various partial waves. The right-top plot emphasizing the angular region where the s-wave contribution is relatively large, the contribution of the $D_{J}\left(j=\frac{1}{2}\right)$ is more visible.

From the analysis, they obtain the mass and the decay width of the new p-wave charm meson: $M_{D_{1}\left(j=\frac{1}{2}\right)}=2.461_{-0.034}^{+0.014}$ (stat) \pm 0.034 (sys) GeV, and $\Gamma_{D_{1}\left(j=\frac{1}{2}\right)}=0.290_{-0.079}^{+0.101}($ stat $) \pm 0.044($ sys $)$ $\mathrm{GeV}$. The product branching fractions of the $B$ decays and $D_{J}$ decays are:

$$
\begin{aligned}
& \mathcal{B}\left(B^{-} \rightarrow D_{1}(2420) \pi^{-}\right) \mathcal{B}\left(D_{1}(2420) \rightarrow D^{*+} \pi^{-}\right) \\
& \left.\quad=6.9_{-1.4}^{+1.8}(\text { stat }) \pm 1.2(\text { sys })\right) \times 10^{-4} \\
& \mathcal{B}\left(B^{-} \rightarrow D_{1}^{0}\left(j=\frac{1}{2}\right) \pi^{-}\right) \mathcal{B}\left(D_{1}^{0}\left(j=\frac{1}{2}\right) \rightarrow D^{*+} \pi^{-}\right) \\
& =10.6 \pm 1.9(\text { stat }) \pm 2.9(\text { sys })) \times 10^{-4},
\end{aligned}
$$

and

$$
\begin{aligned}
& \mathcal{B}\left(B^{-} \rightarrow D_{2}^{*}(2460) \pi^{-}\right) \mathcal{B}\left(D_{2}^{*}(2460) \rightarrow D^{*+} \pi^{-}\right) \\
& =3.1 \pm 0.9(\text { stat }) \pm 0.5(\text { sys })) \times 10^{-4}
\end{aligned}
$$

\section{References}

[1] Over the entire momentum-transfer range $\left(q^{2}\right)$, the ratios among hilicity amplitudes have been measured assuming the Heavy Flavour Effective Theory-based $q^{2}$ dependence.

[2] J.E. Duboscq el al., iPhys. Rev. Lett. 76 (1996), 3898

[3] J.L. Rosner, iPhys. Rev. D $\mathbf{4 2}$ (1990) 3732, M. Neubert, 2 Phys. Lett. B_264 (1991)_455 G. Kramer et al., 'Z. Physik C $\mathbf{5} 5$ (1992) 497,' A. Dighe, I. Duniez and R. Fleischer, hep-ph/9804253'.

[4] S. Pappas, Recent b-Physics Results from CDF, in the Proceedings of the DPF conference, California, USA, Jan 1999.

[5] C.P. Jessop el al., Phys. Rev. Lett. $\mathbf{7 9}(1997)$ 4533

[6]_D. Buskulic el al., 'Phys. Lett. B $\mathbf{3 8 8}(1996))_{1}^{\prime}$ 648

[7] T.E. Coan el al., 'Phys. Rev. Lett. 80 (1998), 1150

[8] R. Barate el al., 'Eur. Phys. J. C 4 (1998) 387'.

[9] P. Abreu el al., 'Phys. Lett. B_426 (1998)_193". 
[10] M.R. Convery, SLAC preprint SLAC-PUB8076, March 1999, contribution to DPF 99, Los Angels, CA, January 1999. hep-ex/9903061'.

_11] M. Artuso el al., 'Phys. Rev. Lett. 82 _(1999)' '- - - 3020!

[12] S. Anderson el al., Contribution to the Lepton Photon Symposium 99, Stanford, CA, August 1999. Cornell Preprint CLEO CONF 99-6. 

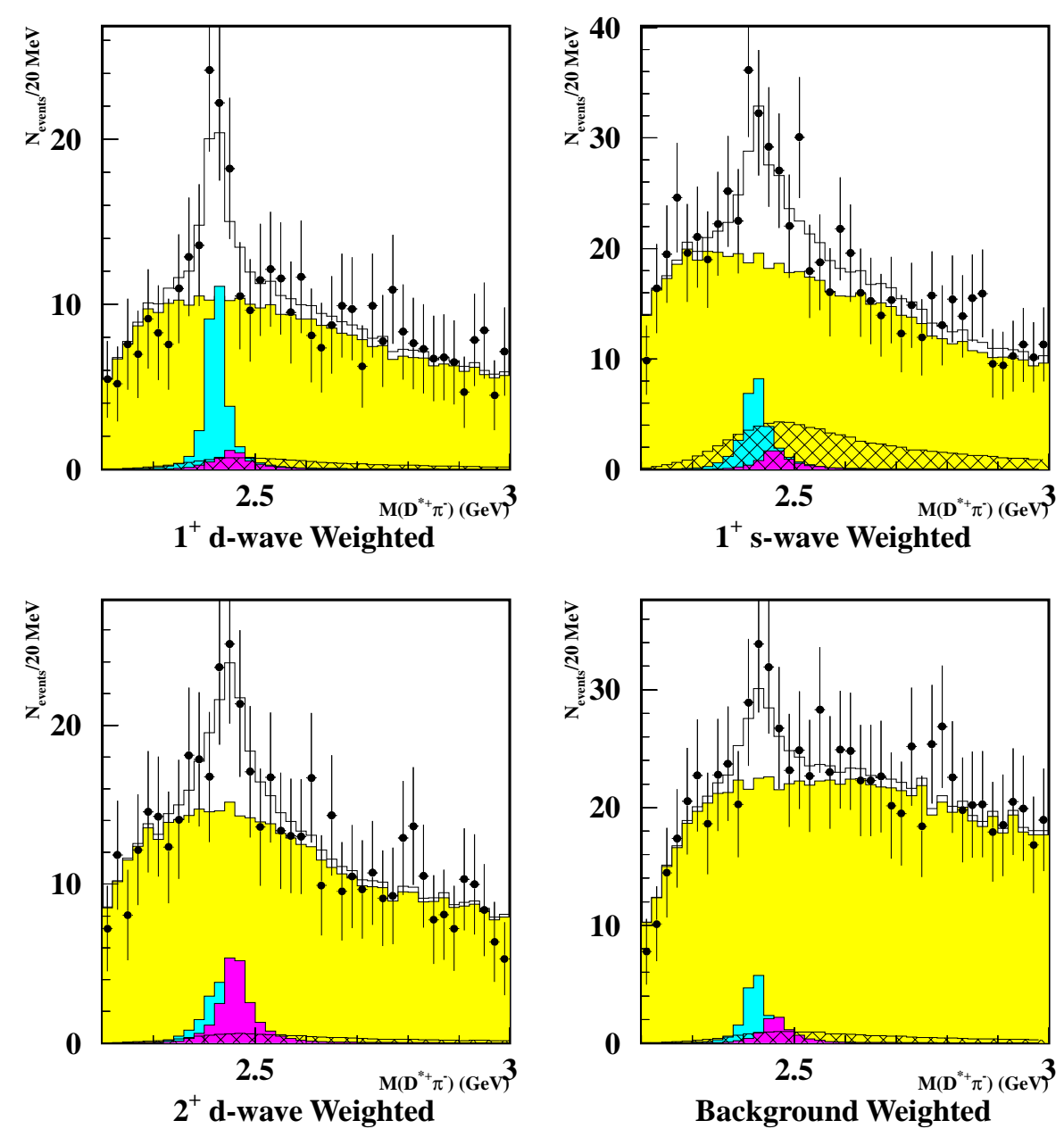

Figure 16: $D^{*+} \pi^{-}$mass distributions with weights based on the decay angles to enhance various partial waves. 